\title{
Quantitative immunocytochemical profile to predict early outcome of disease in triple-negative breast carcinomas
}

\author{
COLETTE CHARPIN $^{1}$, SOPHIE GIUSIANO ${ }^{1}$, VÉRONIQUE SECQ ${ }^{1}$, \\ SÉVERINE CARPENTIER ${ }^{1}$, LUCILE ANDRAC ${ }^{1}$, MARIE-NOËLLE LAVAUT ${ }^{1}$, \\ CLAUDE ALLASIA $^{1}$, PASCAL BONNIER ${ }^{2}$ and STÉPHANE GARCIA $^{1}$ \\ ${ }^{1}$ Department of Pathology, Hôpital Nord and Université de la Méditerranée; \\ ${ }^{2}$ Department of Gynecologic Oncology, Hôpital de la Conception, Marseille, France
}

Received October 30, 2008; Accepted January 9, 2009

DOI: 10.3892/ijo_00000224

\begin{abstract}
We aimed in this study at identifying prognostic immunohistochemical molecular signatures indicative of disease outcome, also relevant for development of new specific therapies, in triple-negative (ER, PR, c-erbB2negative) breast carcinoma subtypes. We evaluated 42 markers in tissue micro-arrays from a series of 924 breast carcinomas including 184 triple-negative tumors using standardized quantitative immunocytochemical assays and correlated the data with patients' outcome (mean follow-up of 79 months). When 27/42 markers including basal-like markers first found to be individually significant for prognosis in a univariate analysis (log-rank test) in 924 tumors, were secondly evaluated in the triple-negative tumor subtype (184/924), eleven including maspin, P21, P27, PTEN, caveolin, EGFR, FAK, P38, pMAPK, STAT1 and CD10 were $89.2 \%$ predictive of disease outcome in logistic regression. When markers reported in the literature as expressed in basal-like subtype were evaluated in the 924 series, only eight (EGFR, CK14, moesin, caveolin, cMet, ckit, CD44v6, C10) were prognosis predictive in univariate analysis (log-rank test) and in logistic regression were predictive of disease outcome in $66.3 \%$ independently of ER, PR and c-erbB2 expression and in $72 \%$ in triple-negative tumor subset. The results suggest that the category of 'triple-negative' breast carcinomas does not exactly overlap the basal-like subtype, and that immunoprofiling of triple-negative tumors (not similar to that of basal-like tumors) may be helpful to select patients for more aggressive treatment and provides a basis for development of tailored therapy.
\end{abstract}

Correspondence to: Professor Colette Charpin-Taranger, Hôpital Nord, Service d'Anatomie et de Cytologie Pathologiques, Chemin des Bourrelly, 13915 Marseille Cedex 20, France

E-mail: colette.charpin@ap-hm.fr

Key words: quantitative immunocytochemistry, tissue micro-arrays, breast carcinomas

\section{Introduction}

Previous studies have reported immunohistochemical profiles of breast carcinomas of various types according the new taxonomic classification based on DNA array profiling, including luminal A and B, Her-2, normal and basal-like carcinoma subtypes. The lack of tailored therapies for the basal-like subtype has led to research interest and clinicopathological studies (1-15).

Likewise, the main concern in routine practice, closely related to academic issues, is the identification of molecules, potentially blockable by new therapies that specifically target them and also indicators of poor prognosis within tumors requiring more aggressive treatment that would be useless in patients lacking such an immunocytochemical profile of poor prognosis, despite being categorized in the same subgroup by clinicopathological criteria (such as small node-negative grade 2 tumors).

In view of the high cost of diagnostic genomic assays and of therapy of breast carcinomas which represent a major public health concern, simpler methods would be desirable to identify proteomic signatures in situ that are predictive of disease outcome. Detection of these signatures by immunohistochemistry would allow selection of tumors to be treated by specific and more aggressive therapies, as an alternative method to the molecular biological approach that is more appropriate to basic and academic purposes. However, such immunocytochemical procedures need to be standardized as far as possible before they can be recommended for routine clinical practice (16-22), preferably using quantification of immunoprecipitates with automated computer-assisted devices based essentially on densitometry, as we (16-22) and others have previously done.

Published data show some small variations in immunohistochemical phenotypes in breast carcinomas (as with DNA assays), probably due to heterogeneity in procedures. Moreover, although molecular and immunohistochemical assays may be dependent on methodological quality (1-15), variations in results can also result from the statistical methods used for data analysis, leading to variable interpretation of biological data (23).

In this study, we aimed, first, to investigate expression of markers reported in the literature in triple-negative tumors 
when evaluated with high-throughput standardized assays using tissue microarrays (TMAs) $(10,13,17,22)$ after validation a large retrospective series $(n=1200)$ of breast carcinomas; second, to quantify immunohistochemical precipitates within digitized microscopic TMA images using an automated computer-assisted device; and third, to correlate the quantified immunohistochemical expression of each marker and of groups of markers, with patients' outcome (mean follow-up 79 months). The overall goal was to identify the best group of markers, in terms of sensitivity and specificity, to predict prognosis in triple-negative tumors within $48 \mathrm{~h}$ on tissue sections, that would be suitable in clinical practice for individual patients at the time of diagnosis, simultaneously with pathological reporting.

\section{Materials and methods}

Patients. The subjects were a consecutive series of 1200 patients with ductal invasive breast carcinoma who were operated on from 1995 to 2002 (mean follow-up 79 months) in the same department (Hôpital Conception, Marseille). Surgery was in all cases the first treatment (PB). For this first step of treatment, patient management was handled by the same group of surgeons and senior pathologists (C.C., S.G. and L.A.). Conservative treatment, mastectomy and node resection (complete or sentinel) were applied according to current European recommendations. Likewise, radiotherapy, chemotherapy and hormone therapy were applied according to criteria currently used at that time.

Analysis of the distribution of the series by age, histological type and grade, and nodal status before TMA construction revealed the usual distribution of breast carcinomas and no bias in tumor selection as previously reported $(10,13,22)$ and as compared to literature data (1-15).

Due to technical difficulties in performing immunocytochemical tests on many serial paraffin sections of a TMA to evaluate the 42 different markers, complete data for all markers were finally obtained for only 924 patients out of the initial series of 1200 .

The 2005 follow-up data in clinical records showed that 181 out of the 924 tumors were metastatic and 32 patients deceased.

Our study focused mainly on correlation of immunohistochemical data with patients' outcome. Current histoprognostic criteria were not further considered for statistical analysis, mainly to limit the burden of data and also to focus the statistical analysis on continuous variables homogeneously obtained by (numerical) densitometric measurements of immunoprecipitates with the image analyser.

Tissues. Tissue samples were all taken from surgical specimens after formalin fixation. Attention was paid to optimal homogeneous tissue handling, including fast immersion in buffered formalin in appropriate containers in the operation room by pathologists or by nurses trained in the procedure. Tumor fragments were large and thick enough to allow further TMA construction. Duration of fixation was $24 \mathrm{~h}$ for smaller samples $(<5 \mathrm{~cm})$ and $48 \mathrm{~h}$ for larger ones, to improve formalin penetration, before specimen dissection at room temperature. After fixation, paraffin pre-embedding and embedding were performed in currently available automated devices of the same brand.

All paraffin blocks were stored in the same room where temperature was maintained at $20^{\circ} \mathrm{C}$ prior to TMA construction.

TMA construction. The procedure for TMA construction was as previously described $(10,13,17,22)$. Briefly, cores were punched from the selected 1200 paraffin blocks (from 1200 patients), distributed in new blocks including two cores for each tumor (200 cases per block, a total of 2400 cores, measuring $0.6 \mathrm{~mm}$ in diameter). All the new blocks were also stored at $4^{\circ} \mathrm{C}$ before sections $4-\mu \mathrm{m}$ thick were prepared for each marker to be examined by immunohistochemical assay.

Immunohistochemistry. Serial tissue sections were assessed and stored at $4^{\circ} \mathrm{C} 24 \mathrm{~h}$ before immunohistochemical processing, as previously reported (16-22). The immunoperoxidase procedure was performed using an automated Ventana Benchmark XT device and Ventana kits.

Markers were detected using commercially available documented antibodies (Table I). Dilutions of antibodies were determined by prescreening on the usual full $4-\mu \mathrm{m}$ thick sections prior to use on TMA sections.

Image analysis. Automated densitometric measurements of immunoprecipitates in cores were assessed for each marker antibody in each core individually identified after digitization and image cropping of the slides, as previously reported $(10,13,22)$. Briefly, TMA analysis with a SAMBA 2050 automated device (SAMBA Technologies, TRIBVN, France) was performed according to the following protocol.

First, an image of the entire slide was built up using a low power magnification ( $\mathrm{x} 2$, pixel dimension $3.7 \mu \mathrm{m}$ ). This image was made of a mosaic of images acquired along a rectangular grid with contiguous fields. Second, the area of the slide containing the TMA cores was automatically delineated and scanned at higher magnification (x10, pixel dimension $7.4 \mu \mathrm{m}$ ). Third, after autofocusing, the images were acquired with an overlap greater than the largest mechanical positioning error. Using the image contents, a matching algorithm determined precisely the relative position of each image with respect to its neighbors. Calculated overlap was removed from images to produce a new set of higher-magnification images (x20), thus covering precisely the cores of interest. A specially developed tool referred to as TMA crop then allowed superimposition of the TMA grid onto the reduced image and precise alignment of each node of the grid with the core location within the image. The final step was performed automatically using the core image contents to ensure pixel precision of the match. From the images acquired with x20 magnification, a new set of images was next computed, one for each core. After color analysis of the core images, the SAMBA 'immuno' software was applied as previously reported (16-22) in the usual full tissue sections.

In the present study, we correlated the patients' follow-up parameters with a quantitative score combining the surface stained and the intensity of staining computed by the Samba ‘immuno' software. 
Table I. The antibodies used in this study.

\begin{tabular}{|c|c|c|c|c|}
\hline & Antibody & Supplier & Source $^{a}$ & Clone \\
\hline 1 & FGFR-1 Flg (C-15) & Santa Cruz & Rpab & FGFR-1 Flg (C-15) \\
\hline 2 & Maspin & BD Pharmingen & Mmab & G167-70 \\
\hline 3 & P-Cadherin & Novocastra & Mmab & $56 \mathrm{C} 1$ \\
\hline 4 & Ezrin (p81, $80 \mathrm{k}$, cytovillin) & Neomarkers & Mmab & $3 \mathrm{C} 12$ \\
\hline 5 & $\mathrm{CD} 44 \mathrm{v} 6$ & Novocastra & Mmab & VFF-7 \\
\hline 6 & Moesin 1 & Biomeda & Mmab & $38 / 87$ \\
\hline 7 & Cytokeratins 8 \& 18 & Zymed & Mmab & Zym5,2(UCD/PR-10,11) \\
\hline 8 & Cytokeratin 17 & Dako & Mmab & E3 \\
\hline 9 & Cytokeratin 14 & Novocastra & Mmab & LL002 \\
\hline 10 & Phospho-STAT3 & Cell Signaling & Mmab & Tyr 705 D3A7 \\
\hline 11 & CD 10 & Novocastra & Mmab & $56 \mathrm{C} 6$ \\
\hline 12 & CD 34 & Dako & Mmab & QBEnd-10 \\
\hline 13 & Vimentin & Immunotech & Mmab & V9 \\
\hline 14 & Cytokeratin 19 & Dako & Mmab & BA17 \\
\hline 15 & Phospho-MAPKAPK-2 & Cell Signaling & Rmab & (Thr334) \\
\hline 16 & EGFR & Ventana & Mmab & $3 C 6$ \\
\hline 17 & STAT-1 & Cell Signaling & Mmab & $9 \mathrm{H} 2$ \\
\hline 18 & FAK & Cell Signaling & Rpab & \\
\hline 19 & p38 MAP kinase & Cell Signaling & Rpab & \\
\hline 20 & P27 Kip1 & Cell Signaling & Rpab & \\
\hline 21 & P21Waf1-Cip1 & Cell Signaling & Mmab & DCS60 \\
\hline 22 & SHARP 2 & Abcam & Rpab & \\
\hline 23 & FYN & Abcam & Mmab & $1 \mathrm{~S}$ \\
\hline 24 & P63 & Dako & Mmab & 4A4 \\
\hline 25 & Cytokeratin 903 & Dako & Mmab & 34BE12 \\
\hline 26 & CA IX & Abcam & Rpab & \\
\hline 27 & E-Cadherin & Zymed & Mmab & $4 \mathrm{~A} 2 \mathrm{C} 7$ \\
\hline 28 & CD 117 (c-Kit) & Dako & Rpab & \\
\hline 29 & Cytokeratins 5-6 & Dako & Mmab & D5/16B4 \\
\hline 30 & PTEN & Cell Signaling & Mmab & $26 \mathrm{H} 9$ \\
\hline 31 & PI3 kinase & Cell Signaling & Rpab & \\
\hline 32 & JAK 1 & Cell Signaling & Rpab & \\
\hline 33 & c-Met & Chemicon/Abcys & Mmab & 4AT44 \\
\hline 34 & Caveolin 1 & Santa Cruz & Rpab & \\
\hline 35 & CD 105 & Dako & Mmab & SN6h \\
\hline 36 & CD 146 & Novocastra & Mmab & N1238 \\
\hline 37 & $\mathrm{Bcl} 2$ & Dako & Mmab & 124 \\
\hline 38 & P53 & Dako & Mmab & DO-7 \\
\hline 39 & P16 & Neomarkers & Mmab & Ab7(16PO7) \\
\hline 40 & c-erbB2 & Novocastra & Mmab & CB11 \\
\hline 41 & PR & Ventana & Mmab & $1 \mathrm{E} 2$ \\
\hline 42 & ER & Ventana & Mmab & $6 \mathrm{~F} 11$ \\
\hline
\end{tabular}

${ }^{\mathrm{a}}$ Mouse monoclonal antibody; rabbit polyclonal antibody.

Statistical analysis. Immunohistochemical expression of each marker was first correlated to patients' disease-free survival using NCSS and Statistica statistical software.

When significant differences in mean expression were identified between patients with and without disease-free survival, the prognostic significance was determined by logrank tests (Kaplan-Meier curves). The appropriate threshold of prognostic significance for a given marker was determined as recommended (24) and as previously assessed in large tissue sections (16-21) and in TMAs $(10,13,22)$. 

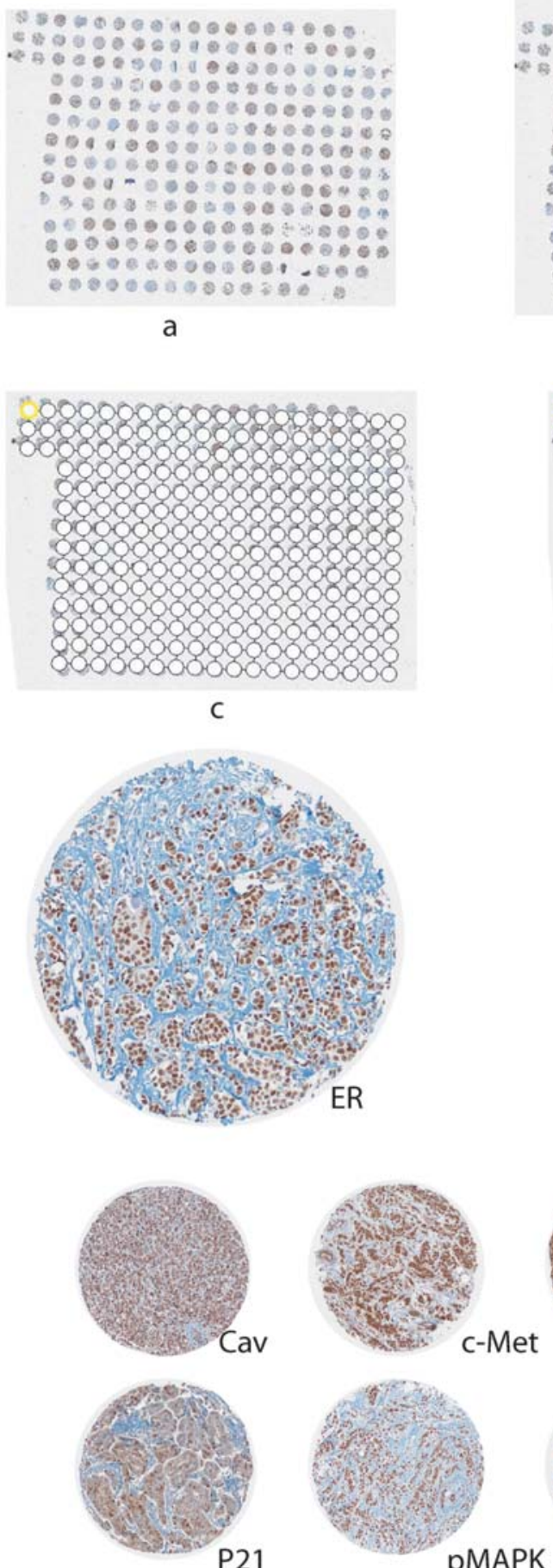

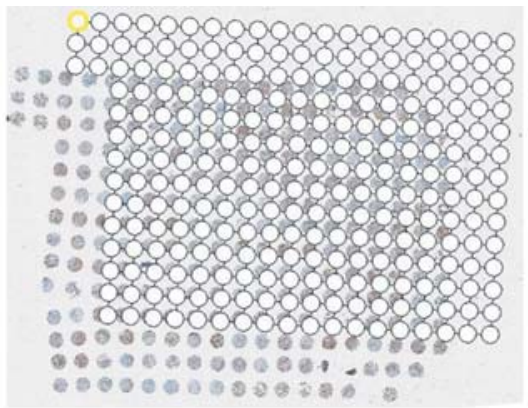

b

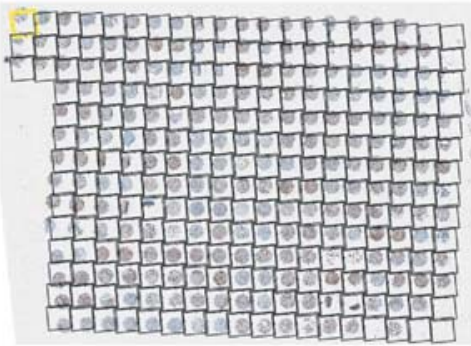

d
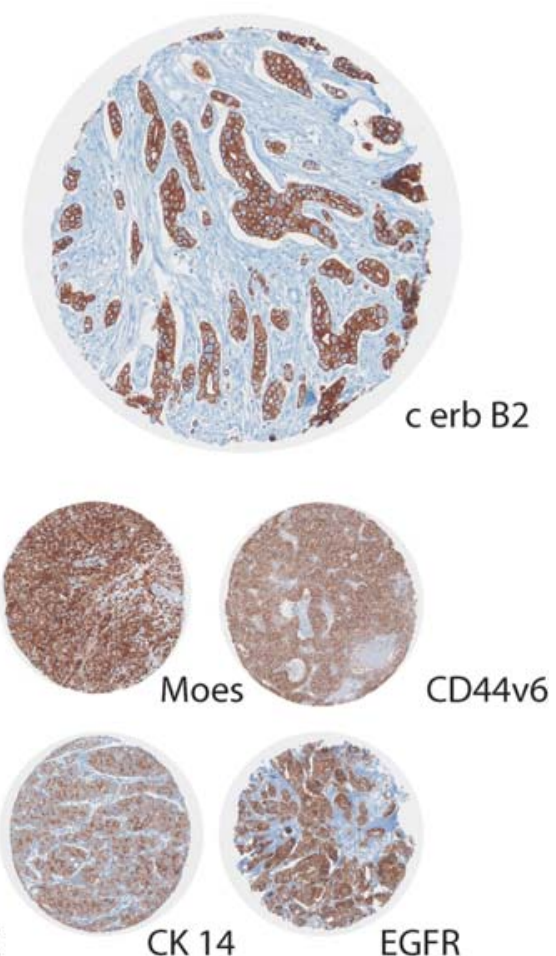

Figure 1. TMA enclosing 268 cores $(0.6 \mu \mathrm{m}$ in diameter) from breast carcinomas: An example of immunohistochemical test and 'cropping' procedure (a, b, c and d) consisting in superimposing a standard grid on the TMA image by a modifying grid shape in order to separate each spot prior to densitometry measurement.

Logistic regression (with ROC curves) was then used to identify the combination of markers with the best sensitivity and specificity of a proteomic signature indicative of prognosis.

Finally, unsupervised hierarchical clustering of significant prognostic indicators in the overall series enabled the relationship of the markers providing qualitative data to be compared with previously reported research results on the role played by these molecules in the process of cancer metastasis.

\section{Results}

Immunostaining in TMAs is illustrated in Fig. 1. Table II shows that 27 markers were prognostically significant in univariate analysis after determination of the cut-off value according to Altman et al (24) (Fig. 2), in 924 tumors for which complete immunocytochemical data could be obtained.

Logistic regression was performed for those markers with prognostic significance in the univariate log-rank analysis in 
Table II. Optimal thresholds of quantitative scores for 27 markers of prognostic significance in univariate analysis log-rank test, as determined according to the method of Altman et al (24) for 924 patients with breast cancer.

\begin{tabular}{|c|c|c|c|}
\hline & & $\begin{array}{l}\text { P-value } \\
\text { (log-rank) }\end{array}$ & $\begin{array}{c}\text { Quantitative score } \\
\text { threshold }\end{array}$ \\
\hline 1 & SHARP 2 & $<0.001$ & 5.6 \\
\hline 2 & PR & $<0.0001$ & 7.9 \\
\hline 3 & ER & $<0.01$ & 9.3 \\
\hline 4 & STAT1 & $<0.0001$ & 10.7 \\
\hline 5 & PTEN & $<0.0001$ & 2.7 \\
\hline 6 & pSTAT-3 & $<0.01$ & 4.4 \\
\hline 7 & pMAPK & $<0.001$ & 12.5 \\
\hline 8 & P38 & $<0.001$ & 1.1 \\
\hline 9 & $\mathrm{P} 27$ & $<0.01$ & 5.3 \\
\hline 10 & $\mathrm{P} 21$ & $<0.001$ & 10.3 \\
\hline 11 & P16 & $<0.01$ & 7.5 \\
\hline 12 & Moesin & $<0.0001$ & 16.4 \\
\hline 13 & Maspin & $<0.001$ & 7.3 \\
\hline 14 & CK14 & $<0.001$ & 8.3 \\
\hline 15 & FYN & $<0.01$ & 2.7 \\
\hline 16 & FGF-R & $<0.01$ & 23.0 \\
\hline 17 & FAK & $<0.001$ & 2.0 \\
\hline 18 & Ezrin & $<0.001$ & 3.9 \\
\hline 19 & c-Kit & $<0.001$ & 12.9 \\
\hline 20 & c-Met 2 & $<0.01$ & 17.0 \\
\hline 21 & EGFR & $<0.01$ & 9.4 \\
\hline 22 & CK19 & $<0.01$ & 4.6 \\
\hline 23 & c-erbB2 & $<0.001$ & 28.0 \\
\hline 24 & CD 44 v6 & $<0.001$ & 11.9 \\
\hline 25 & CD 146 & $<0.001$ & 2.3 \\
\hline 26 & CD 10 & $<0.01$ & 4.0 \\
\hline 27 & Caveolin & $<0.01$ & 29.0 \\
\hline
\end{tabular}

patients with ER-, PR- and c-erbB2-negative tumors ( $n=184 / 924)$, of which 168/181 were disease-free and 16/181 metastatic.

The first-step regression showed that with 24 markers (Table III) (Fig. 2a and b), 89.7\% of the patients were well classified (15/16 metastatic patients with the 24-marker signature and 18/168 disease-free patients with the 24-marker signature), with $93.8 \%$ sensitivity and $89.3 \%$ specificity. In a second-step regression using an 11-marker signature including EGFR, caveolin, FAK, maspin, P21, P27, PTEN, P38, pMAPK, STAT-1 and CD10 (Table IV and Fig. 3), 89.1\% of patients were well classified.

Unsupervised hierarchical clustering of the 24 prognostic markers is shown in Fig. 4 (independently of ER, PR and cerbB2 status) (Fig. 4).

Eight markers of the basal-like subtype (c-Met, caveolin, moesin, CD44v6, CK14, EGFR, c-Kit and CD10) reported in the literature (1-15) and predictive of disease outcome on log-rank univariate analysis in the series of 924 patients were evaluated by logistic regression (Table V, Fig. 3) independently
Table III. Logistic regression of 24 markers of prognostic significance in triple-negative tumors $(n=184 / 924)$ in univariate log-rank test.

\begin{tabular}{|c|c|c|}
\hline & Immunocytochemical marker & P-value \\
\hline 1 & c-Kit & 0.068 \\
\hline 2 & CD10 & 0.0001 \\
\hline 3 & STAT-1 & 0.0003 \\
\hline 4 & SHARP2 & 0.44297 \\
\hline 5 & PTEN & 0.0238 \\
\hline 6 & pSTAT3 & 0.56922 \\
\hline 7 & pMAPK & 0.00224 \\
\hline 8 & P38 & 0.04678 \\
\hline 9 & $\mathrm{P} 27$ & 0.0077 \\
\hline 10 & $\mathrm{P} 21$ & 0.00015 \\
\hline 11 & P16 & 0.1621 \\
\hline 12 & Moes & 0.0559 \\
\hline 13 & Maspin & 0.00251 \\
\hline 14 & CK14 & 0.11288 \\
\hline 15 & FYN & 0.40538 \\
\hline 16 & FgFR1 & 0.99544 \\
\hline 17 & FAK & 0.00974 \\
\hline 18 & EGFR & 0.0782 \\
\hline 19 & c-Met & 0.56346 \\
\hline 20 & CK19 & 0.26917 \\
\hline 21 & CD44v6 & 0.54556 \\
\hline 22 & CD146 & 0.39627 \\
\hline 23 & CD10 & 0.0088 \\
\hline 24 & Caveolin & 0.01813 \\
\hline
\end{tabular}

Table IV. Logistic regression in triple-negative $(n=184 / 924)$ tumors with 11 markers.

\begin{tabular}{rll}
\hline & Immunocytochemical marker & P-value \\
\hline 1 & STAT1 & 0.024 \\
2 & PTEN & 0.0422 \\
3 & pMAPK & 0.02568 \\
4 & P38 & 0.04002 \\
5 & P27 & 0.00747 \\
6 & P21 & 0.00022 \\
7 & Maspin & 0.00543 \\
8 & CD10 & 0.00061 \\
9 & FAK & 0.01148 \\
10 & EGFR & 0.0018 \\
11 & Caveolin & 0.04868 \\
\hline
\end{tabular}

of ER, PR and c-erbB2 status. The first-step regression showed that $66.3 \%$ (69.6\% sensitivity and $65.5 \%$ specificity) of the patients were well classified, including 126/181 metastatic patients (with the eight-marker signature) and 218/743 disease-free patients (without the eight-marker 

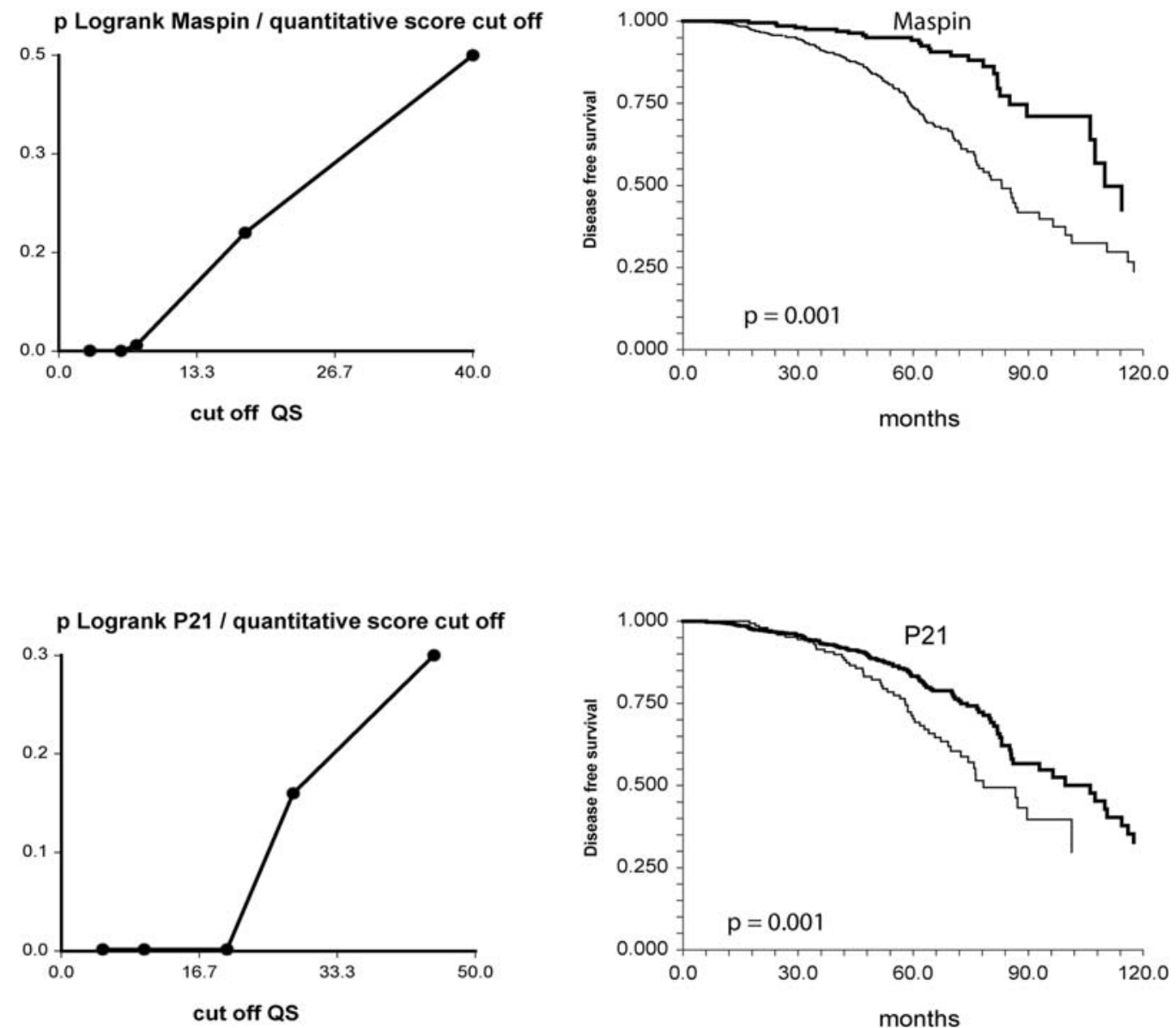

Figure 2. Kaplan-Meier curves (log-rank test) and p-value curves [according to Altman et al (24)] used to determine the threshold of quantitative scores for prognostic significance of markers; in this example, P21 and maspin in 924 breast carcinomas (TMA, quantitative immunochemical assays).

Table V. Logistic regression of eight basal-like markers predictive of prognosis in log-rank univariate analysis in 924 breast carcinomas.

\begin{tabular}{llc}
\hline & Immunocytochemical marker & P-value \\
\hline 1 & EGFR & 0.09417 \\
2 & CK-14 & 0.00119 \\
3 & Moesin & 0.00001 \\
4 & Caveolin & $<0.0001$ \\
5 & c-Met & 0.00169 \\
6 & cKIT & 0.00118 \\
7 & CD44v6 & 0.00187 \\
8 & CD10 & 0.00577 \\
\hline
\end{tabular}

signature). In a second-step regression with seven markers (excluding EGFR), a similar $64.8 \%$ of patients were well classified (Table V, Fig. 3).

When markers of basal-like carcinomas were considered in triple-negative tumors, the first step of logistic regression with 11 markers showed that $72 \%$ of patients were well classified and in a second step regression with 10 markers, 71.6\% were well classified (Tables VI and VII and Fig. 3).
Table VI. Logistic regression with basal-like markers that were prognostically predictive in univariate log rank test, when ER, PR and c-erbB2 are included in the regression in 924 breast carcinomas.

\begin{tabular}{rll}
\hline & Immunocytochemical markers & P-value \\
\hline 1 & Caveolin & 0.0002 \\
2 & CD44v6 & 0.03555 \\
3 & c-erbB2 & 0.0002 \\
4 & c-KIT & 0.00101 \\
5 & c-Met & 0.00012 \\
6 & EGFR & 0.04559 \\
7 & CK14 & 0.00204 \\
8 & Moesin & 0.00002 \\
9 & CD10 & 0.00388 \\
10 & ER & 0.00888 \\
11 & PR & $<0.0001$ \\
\hline
\end{tabular}

\section{Discussion}

Immunocytochemical signatures of triple-negative and basal-like tumors. Several published studies (1-15) have 
a

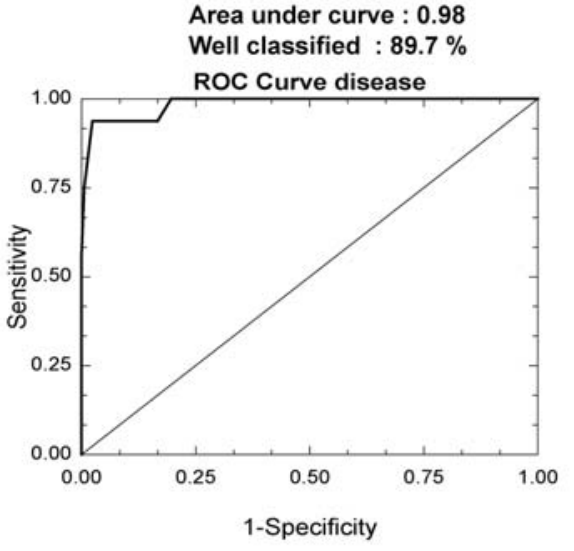

c

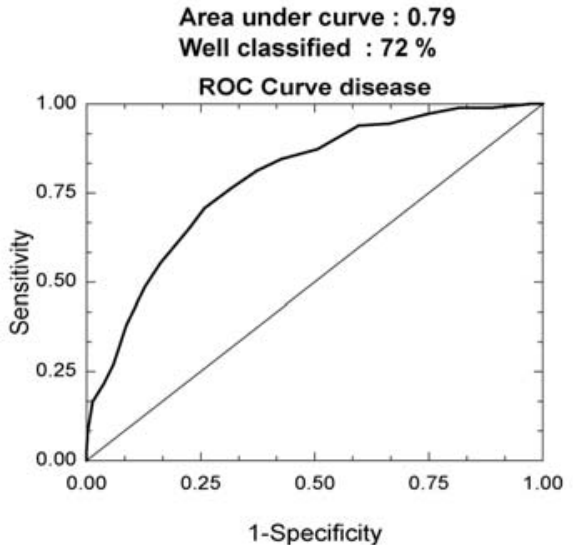

e

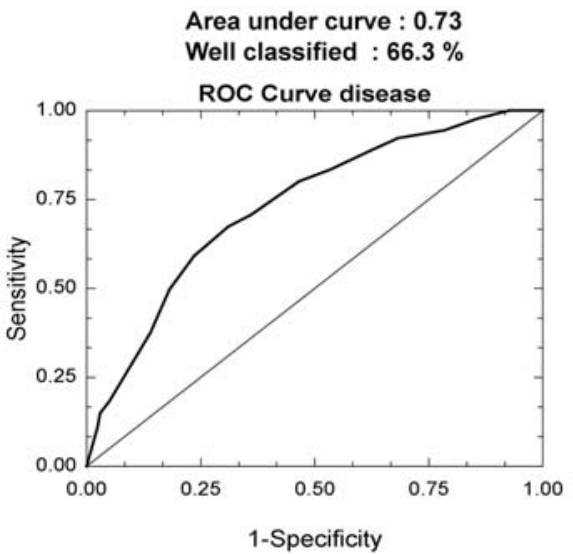

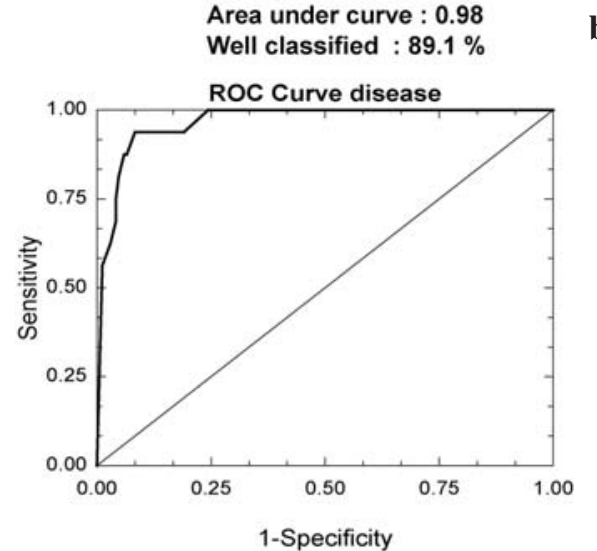

b

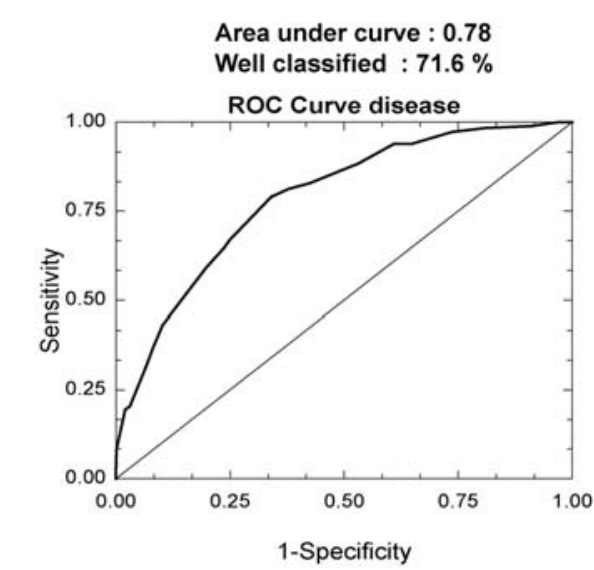

d

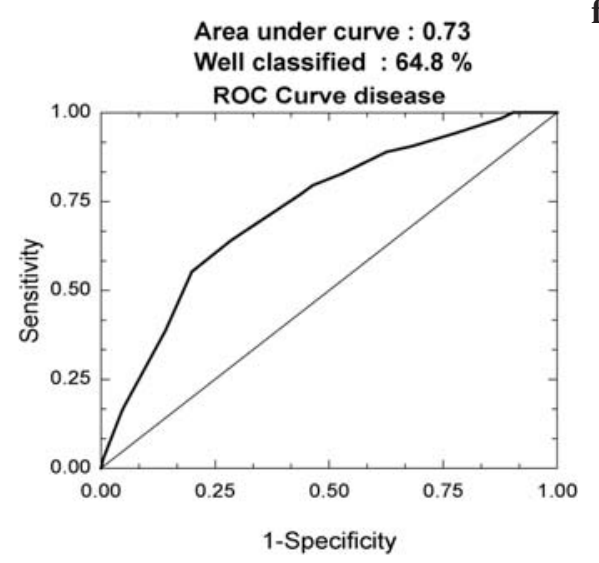

f

Figure 3. Logistic regression and ROC curves first (a, c, e) and second (b, d, f) steps: (a) with 24 and (b) with 11-marker signatures in triple-negative tumors $(\mathrm{n}=184)$ ( 89.7 and $89.1 \%$ well classified patients); (c) with 8 and (d) 7 basal-like markers in triple-negative tumors (72 and $71.6 \%)$ well classified patients; and (e) 8 and (f) 7 basal-like markers in the complete 924 series (66.3 and $64.8 \%$ well classified patients).

documented basal-like carcinomas that represent a subtype of breast carcinomas lacking tailored therapies, since they most often lack ER and PR receptors and c-erbB2 amplification. However, some recent studies $(7,8)$ have shown that triplenegative tumors may not be entirely equivalent to basal-like carcinomas. Although exhibiting immunoprofiles closely similar to that reported for the basal-like subtype, triplenegative carcinomas respond to anthracyclines but, like BRCA1 tumors, have lower sensitivity to taxans, are very aggressive tumors with early recurrence and short survival (8) and also have epithelial mesenchymal transition or basal myoepithelial profiles (25-27). In fact, these triple-negative tumors lack an internationally accepted definition, which will depend upon the thresholds used for ER- and PR-negativity.

In our study, we used quantitative immunohistochemical assays to identify a signature giving $89.1 \%$ prediction of poor prognosis and early recurrence with 11 markers in triplenegative tumors. The ER- and PR-positive status was defined according to the quantitative score threshold for ER and PR prognostic value in a log-rank univariate analysis, after validation of cut-off points using the curve of p-values according to the recommendations of Altman et al (24), as shown in Table II and Fig. 2. This 11-marker signature (out of 24 including markers of basal-like subtype and others) 

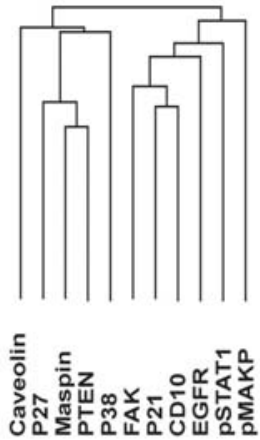
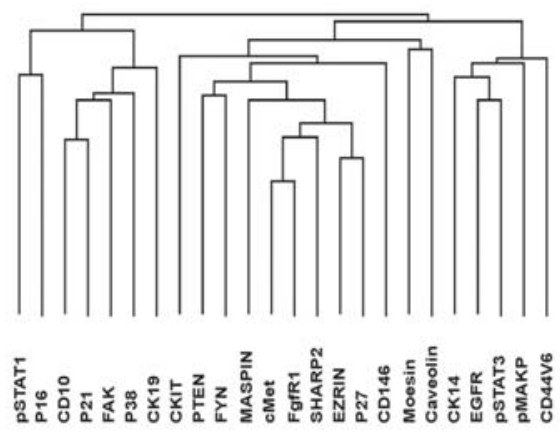
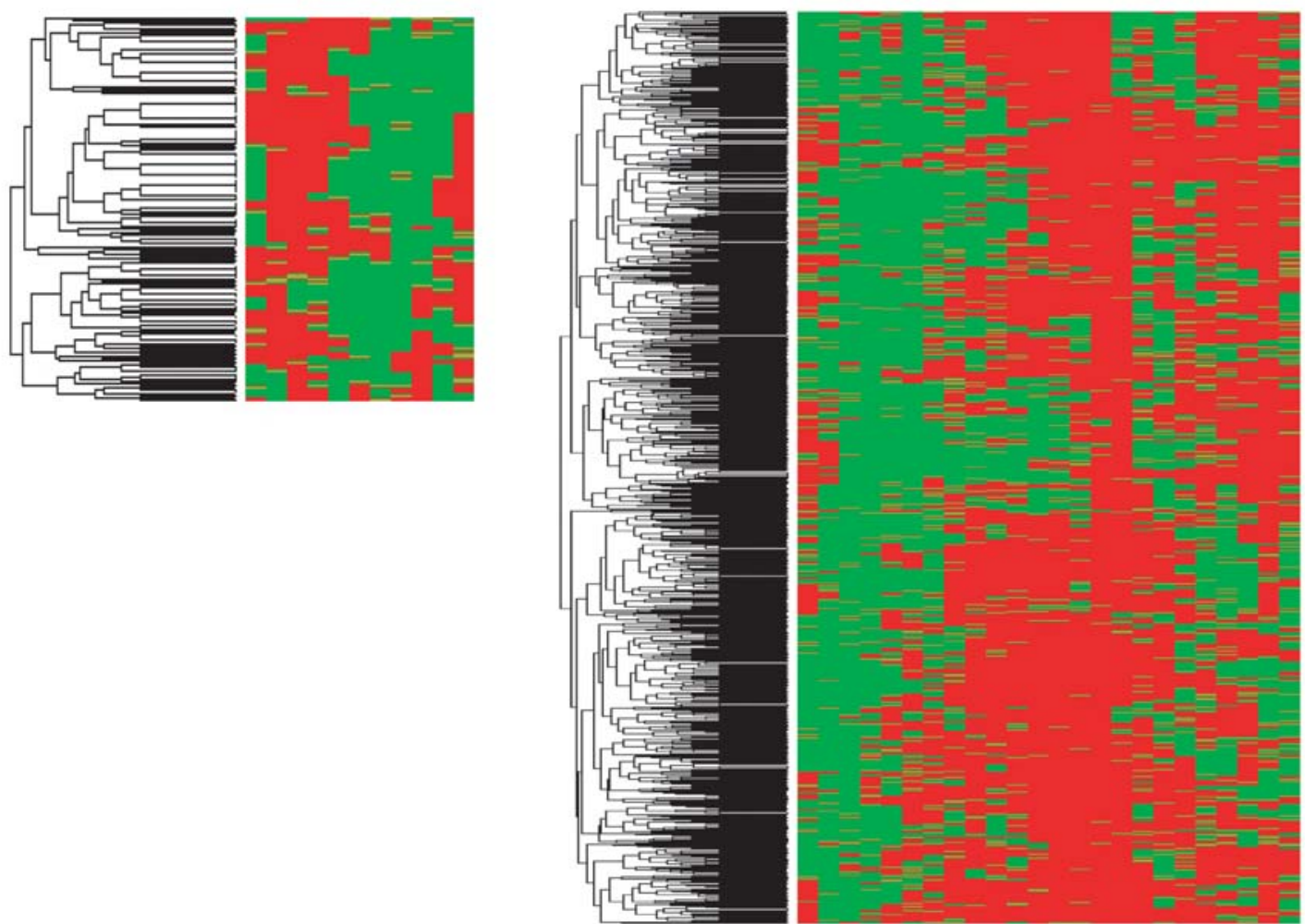

Figure 4. Unsupervised hierarchical clustering of the 24 markers with prognostic significance in the log-rank test, from quantitative densitometry of immunohistochemical assays on TMA in the 924 series of patients with breast carcinoma and of the 11 markers prognostic significant in the 184 triple-negative carcinomas.

Table VII. Summary of logistic regression with immunoprofiling of basal-like tumors in 924 breast carcinomas.

Signatures/Immunocytochemical Sensitivity Specificity Well classified Number of patients well classified $(\mathrm{n}=924)$ markers of prognosis

$\% \quad \overline{\frac{\text { Positive }}{\%}}$

Negative and no events

'Basal-like' without ER,

$\mathrm{PR}, \mathrm{c}-\mathrm{erbB} 2^{\mathrm{a}}$ ( $\mathrm{n}=8$ markers)

$\begin{array}{llll}69.6 & 65.5 & 66.3 & 126 / 181 \\ 74.6 & 62.45 & 64.8 & 135 / 181 \\ & & & \\ 70.66 & 74.6 & 72.0 & 135 / 181 \\ 71.3 & 71.7 & 71.6 & 129 / 181\end{array}$

$256 / 743$

Second regression $(n=7)$

69.6

'Basal-like'with ER,

PR, c-erbB2a ( $\mathrm{n}=11$ markers)

71.7

$129 / 181$

Second regression $(\mathrm{n}=10)$

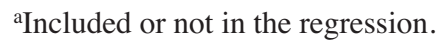


included caveolin, EGFR, FAK, maspin, P21, P27, PTEN, P38, pMAPK, STAT-1, CD10 (plus negative ER, PR, cerbB2) (Table IV). When only basal-like markers, selected according to literature data and prognostically significant in univariate analysis of our series, were included in the logistic regression to predict disease outcome, $72 \%$ of patients were well classified with 8 markers including EGFR, c-Kit, moesin, caveolin, CD10, c-Met, CD44v6, in ER-, PRand c-erbB2-negative tumors (Table IV). This result suggests that categories of triple-negative and basal-like tumors do not completely overlap, in terms of the quantitative immunocytochemical assays that we used, as previously shown (8).

Prognostic value of markers in signatures. Marker proteins involved in tumor cell motility and spreading were of particular interest in our study to predict patients' outcome, which is highly dependent on development of metastasis. Some studies have underlined the role of c-Met in tumor spreading (reviewed in ref. 10). We and others have shown the significance of c-Met for poor prognosis in breast cancers $(10,21,22)$ and also that of CD44v6 (28).

Recent studies have shown the synergistic role of CD44v6 and c-Met in several types of tumor cells (28) and the interaction of the EMR (ezrin-moesin-radixin) superfamily and CD44v6 for HGF activation of c-Met to promote the ERK signalling cascade, inducing cell migration. Moreover, EMR components act on cell adhesion (integrin B2) and the cytoskeleton $(29,30)$. FAK (focal adhesion kinase) is also known to play a pivotal role in the control of integrin-mediated cell functions including cell migration, progression and survival, co-acting with c-Met and EMR (31). Consistent with these findings, CD44v6, c-Met, moesin and FAK are included in the immunocytochemical signature of poor prognosis determined by logistic regression for early (79 months) metastatic disease in basal-like carcinomas, but not in triple-negative tumors.

Cytokeratin 14 expression in association with EGFR in basal-like carcinomas has already been shown to be a strong indicator of poor outcome and particularly predictive in relation to lung and brain metastases. Our results correlate with these previous studies $(6,7,26,27)$.

CK14 is found in metaplastic carcinomas in association with P63 and EGFR $(25,26)$. CK14 was a significant indicator of risk of metastasis in our study in basal-like carcinomas but not in triple-negative tumors. CK5-6 with CD10 was also previously shown to be a prognostic indicator (5).

Caveolins are membrane proteins involved in membrane trafficking, gene regulation, signal transduction and mediation of intracellular processes, as well as in carcinogenesis, being overexpressed in 4 to $57 \%$ of invasive breast carcinomas $(32,33)$. Conflicting results on the prognostic significance of overexpression in breast carcinomas have been reported (32). More specifically, caveolin has been reported in basal-like and metaplastic breast carcinomas (26,32). Interestingly, our results show that caveolin in invasive breast carcinomas is definitely associated with poor prognosis in univariate analysis and in triple-negative tumors and along with markers of tumor cell spreading (and increased motility) in basal-like tumors after logistic regression.
High-grade breast tumors with reduced expression of tumor-suppressor genes such as PTEN and P21 and P27, are associated with poor outcome. In our study, reduced expression of P21 and P27 showed prognostic significance in logistic regression in triple-negative tumors and are probably also predictors of responsiveness to chemotherapy.

Prediction of reponse to therapy and immunocytochemical profiling. New approaches to molecular typing of tumors are relevant to prognosis, but also to prediction of response to therapy. Therefore, markers identified as prognostic indicators can also be regarded as indicators of responsiveness to current chemotherapies or as targets for tailored therapies. For example, caveolins, moesin and CD44v6 have been shown to be indicators of responsiveness to anthracyclines and paxitaxel (8). Also, caveolins have been shown to be predictors of response to platinum salts (reviewed in ref. 8).

Caveolins have recently been shown to be overexpressed and amplified in a subset of basal-like and metaplastic breast carcinomas $(3,5,32)$. Savage et al (32) showed that caveolin 1 expression was significantly associated with basal-like subtype and with shorter disease-free and overall survival on univariate analysis. ABI-007 is a novel, biologically interactive, nanometer-sized albumin-bound paclitaxel particle initially developed to avoid toxicity associated with polyethylated castor oil (34). In a phase III study, Gradishar et al (34) compared ABI-007 with polyethylated castor oilbased paclitaxel in women with metastatic breast cancer. ABI007 showed greater efficacy and a favorable safety profile, though no subgroup analysis of molecular phenotypes for differential efficacy of the treatment was performed. After incorporation of ABI-007 with albumin into the blood circulation, ABI-007 is preferentially transported from the blood to the tumor site in two ways. One route is through leaky junctions of endothelial cells that are highly pronounced around the tumor tissue by induction of angiogenesis. The second, perhaps more prominent, way is through receptor-mediated transcytosis of this albuminbound ABI-007. This second mechanism is mediated by caveolin. Breast cancer patients with higher caveolin 1 expression may therefore show better efficacy and a more favorable safety profile when treated with ABI-007 (35).

Moesin has already been shown to be overexpressed in breast cancer of poor prognosis such as the basal-like subtype (5). Our study has confirmed this finding in a large series, showing in both univariate analysis and logistic regression that moesin has prognostic significance in tumors expressing other basal-like markers, but not in triple-negative ones.

Dasatinib is a small molecule orally active as a kinase inhibitor of both the src and abl proteins. Finn et al (36) recently reported that it selectively inhibits growth of basallike and 'triple-negative' breast cancer cell lines growing in vitro. Interestingly, they identified a set of three biologically relevant genes whose elevated expression is associated with dasanitib inhibition, including moesin, caveolins and yesassociated protein, with sensitivity and specificity of 88 and $86 \%$, respectively. Therefore dasatinib may also be an effective treatment option in breast cancer subtypes with caveolin expression (36). Dasananib is also active against PDGF-R and c-Kit and has been shown to be efficient in 
leukemia after failure of imatinib therapy (37). However, response to imatinib and dasatinib in breast carcinomas with c-Kit expression remains to be demonstrated. Likewise, sunitinib, that is recommended in gastrointestinal stromal tumors (GIST) expressing c-Kit and in advanced kidney carcinomas, is an inhibitor of angiogenic tyrosine kinase receptors involved in angiogenesis such as PDGR, VEGFR, FLT3 and c-Kit. This suggests that c-Kit in breast carcinomas of poor prognosis could also be targeted by sunitinib.

Antibodies against c-Met and small molecules such as PHA66752 or kerin that target c-Met, or the NK4 molecule that blocks HGF binding to c-Met have been reported (reviewed in ref. 10) to act as specific inhibitors of c-Met and signalling pathways including P38, pMAPK, ERK, FAK and P21 in breast cancer, while other tyrosine kinase inhibitors such as Iressa, Tarceva, Herceptin and Genifinib do not inhibit c-Met activity (38).

EGFR is expressed in tumors of poor prognosis and has been included in the signature for poor prognosis of triplenegative and basal-like carcinomas $(1,2-9,11,12,15)$. Interestingly, we also observed that significantly increased expression of the STAT-3 and STAT-1 signalling pathway, resulting partly from EGFR activation, was associated with poor outcome, like others (reviewed in ref. 8), suggesting that EGFR is activated when immunocytochemically overexpressed.

Malignant EGFR-expressing tumors have been shown to respond to tailored therapies like cetuximab (a monoclonal antibody against EGFR), lapatinib (an EGFR tyrosine kinase receptor inhibitor), or gefitinib or enlotinib, which are inhibitors of cellular kinase activity. Although cetuximab has been shown to reduce death in laryngeal tumors and gefitinib and enlotinib in non-small cell lung cancer (8), no data are available on levels of EGFR expression in breast carcinomas and prediction of tumor responsiveness to specific therapy. But new tools are now available to rapidly detect amplification of the c-erbB2 gene by FISH/CISH/SISH so as to better identify tumors potentially responsive to these specific therapies.

In conclusion, using quantitative standardized immunocytochemical assays, we measured some established markers of basal-like breast carcinomas, such as EGFR, CK14, c-Met, caveolins, moesin, CD44v6, c-Kit, CD10, that individually are predictive of disease outcome in log-rank univariate analysis and together are $66.3 \%$ predictive of poor prognosis, but are $72 \%$ predictive when associated with ER-, PR- and c-erbB2-negative status. When triple-negative tumors were considered among 24 markers found to be individually prognostically significant in univariate log-rank analysis, the association of 11 markers (EGFR, maspin, P21, P27, PTEN, P38, pMAPK, STAT-1, caveolin, FAK and CD10) was $89.2 \%$ predictive of poor prognosis and probably indicative of responsiveness of current chemotherapy. Our results suggest that i) triple-negative and basal-like tumors are not identical types, since their prognostic immunoprofiles do not overlap and ii) the immunomolecular signatures of both of these types may be helpful in current practice to select patients for more aggressive therapy and to develop new, more specific targeted therapies.

\section{Acknowledgements}

Supported by a grant from the Institut National contre le Cancer/Canceropole Provence Alpes Côte d'Azur-Assistance Publique-Hôpitaux de Marseille. We are grateful to $\mathrm{ROCHE}$ for financial support of Master ant $\mathrm{PhD}$ (S. Giusiano, V. Secq).

\section{References}

1. Ginestier C, Charafe-Jauffret E, Bertucci F, et al: Distinct and complementary information provided by use of tissue and DNA microarrays in the study of breast tumor markers. Am J Pathol 161: 1223-1233, 2002.

2. Nielsen TO, Hsu FD, Jensen $\mathrm{K}$, et al: Immunohistochemical and clinical characterization of the basal-like subtype of invasive breast carcinoma. Clin Cancer Res 10: 5367-5374, 2004.

3. Charafe-Jauffret E, Ginestier C, Monville F, et al: Gene expression profiling of breast cell lines identifies potential new basal markers. Oncogene 25: 2273-2284, 2006.

4. Livasy CA, Karaca G, Nanda R, et al: Phenotypic evaluation of the basal-like subtype of invasive breast carcinoma. Mod Pathol 19: 264-271, 2006.

5. Charafe-Jauffret E, Monville F, Bertucci F, et al: Moesin expression is a marker of basal breast carcinomas. Int $\mathrm{J}$ Cancer 121: 1779-1785, 2007.

6. Laakso M, Tanner M, Nilsson J, et al: Basoluminal carcinoma: a new biologically and prognostically distinct entity between basal and luminal breast cancer. Clin Cancer Res 12: 4185-4191, 2006.

7. Da Silva L, Clarke C and Lakhani SR: Demystifying basal-like breast carcinomas. J Clin Pathol 60: 1328-1332, 2007.

8. Reis-Filho JS and Tutt AN: Triple negative tumours: a critical review. Histopathology 52: 108-118, 2008.

9. Rakha EA, El-Sayed ME, Green AR, Paish EC, Lee AH and Ellis IO: Breast carcinoma with basal differentiation: a proposal for pathology definition based on basal cytokeratin expression. Histopathology 50: 434-438, 2007.

10. Garcia S, Dales JP, Charafe-Jauffret E, et al: Poor prognosis in breast carcinomas correlates with increased expression of targetable CD146 and c-Met and with proteomic basal-like phenotype. Hum Pathol 38: 830-841, 2007.

11. Korsching E, Packeisen J, Agelopoulos K, et al: Cytogenetic alterations and cytokeratin expression patterns in breast cancer: integrating a new model of breast differentiation into cytogenetic pathways of breast carcinogenesis. Lab Invest 82: 1525-1533, 2002.

12. Makretsov NA, Huntsman DG, Nielsen TO, et al: Hierarchical clustering analysis of tissue microarray immunostaining data identifies prognostically significant groups of breast carcinoma. Clin Cancer Res 10: 6143-6151, 2004.

13. Garcia S, Dales JP, Jacquemier J, et al: c-Met overexpression in inflammatory breast carcinomas: automated quantification on tissue microarrays. Br J Cancer 96: 329-335, 2007.

14. Fulford LG, Easton DF, Reis-Filho JS, et al: Specific morphological features predictive for the basal phenotype in grade 3 invasive ductal carcinoma of breast. Histopathology 49: 22-34, 2006.

15. Jacquemier J, Ginestier C, Rougemont J, et al: Protein expression profiling identifies subclasses of breast cancer and predicts prognosis. Cancer Res 65: 767-779, 2005.

16. Charpin C, Garcia S, Bouvier C, et al: CD31/PECAM automated and quantitative immunocytochemical assays in breast carcinomas: correlation with patient follow-up. Am J Clin Pathol 107: 534-541, 1997.

17. Charpin C, Dales JP, Garcia S, et al: Tumor neoangiogenesis by CD31 and CD105 expression evaluation in breast carcinoma tissue microarrays. Clin Cancer Res 10: 5815-5819, 2004.

18. Charpin C, Garcia S, Bonnier P, et al: Prognostic significance of Nm23/NDPK expression in breast carcinoma, assessed on 10-year follow-up by automated and quantitative immunocytochemical assays. J Pathol 184: 401-407, 1998.

19. Charpin C, Garcia S, Bonnier P, et al: bcl-2 automated and quantitative immunocytochemical assays in breast carcinomas: correlation with 10-year follow-up. J Clin Oncol 16: 2025-2031, 1998. 
20. Charpin C, Vielh P, Duffaud F, et al: Quantitative immunocytochemical assays of P-glycoprotein in breast carcinomas: correlation to messenger RNA expression and to immunohistochemical prognostic indicators. J Natl Cancer Inst 86: 1539-1545, 1994.

21. Charpin C, Garcia S, Bouvier C, et al: Automated and quantitative immunocytochemical assays of CD44v6 in breast carcinomas. Hum Pathol 28: 289-296, 1997.

22. Garcia S, Dales JP, Charafe-Jauffret E, et al: Overexpression of c-Met and of the transducers PI3K, FAK and JAK in breast carcinomas correlates with shorter survival and neoangiogenesis. Int J Oncol 31: 49-58, 2007.

23. Michiels S, Koscielny S and Hill C: Prediction of cancer outcome with microarrays: a multiple random validation strategy. Lancet 365: 488-492, 2005.

24. Altman DG, Lausen B, Sauerbrei W and Schumacher M: Dangers of using 'optimal' cutpoints in the evaluation of prognostic factors. J Natl Cancer Inst 86: 829-835, 1994.

25. Reis-Filho JS, Milanezi F, Steele D, et al: Metaplastic breast carcinomas are basal-like tumours. Histopathology 49: 10-21, 2006.

26. Rakha EA, Putti TC, Abd El-Rehim DM, et al: Morphological and immunophenotypic analysis of breast carcinomas with basal and myoepithelial differentiation. J Pathol 208: 495-506, 2006.

27. Sarrio D, Rodriguez-Pinilla SM, Hardisson D, Cano A, Moreno-Bueno $\mathrm{G}$ and Palacios J: Epithelial-mesenchymal transition in breast cancer relates to the basal-like phenotype. Cancer Res 68: 989-997, 2008.

28. Orian-Rousseau V, Morrison H, Matzke A, et al: Hepatocyte growth factor-induced Ras activation requires ERM proteins linked to both CD44v6 and F-actin. Mol Biol Cell 18: 76-83, 2007.

29. Niggli V and Rossy J: Ezrin/radixin/moesin: versatile controllers of signaling molecules and of the cortical cytoskeleton. Int J Biochem Cell Biol 40: 344-349, 2008.
30. Tang $\mathrm{P}, \mathrm{Cao} \mathrm{C}, \mathrm{Xu} \mathrm{M}$ and Zhang L: Cytoskeletal protein radixin activates integrin alpha(M)beta(2) by binding to its cytoplasmic tail. FEBS Lett 581: 1103-1108, 2007.

31. Chen SY and Chen HC: Direct interaction of focal adhesion kinase $(\mathrm{FAK})$ with Met is required for FAK to promote hepatocyte growth factor-induced cell invasion. Mol Cell Biol 26: 5155-5167, 2006.

32. Savage K, Lambros MB, Robertson D, et al: Caveolin 1 is overexpressed and amplified in a subset of basal-like and metaplastic breast carcinomas: a morphologic, ultrastructural, immunohistochemical, and in situ hybridization analysis. Clin Cancer Res 13: 90-101, 2007.

33. Liedtke C, Kersting C, Burger H, Kiesel L and Wulfing P: Caveolin-1 expression in benign and malignant lesions of the breast. World J Surg Oncol 5: 110-111, 2007.

34. Gradishar WJ, Tjulandin S, Davidson N, et al: Phase III trial of nanoparticle albumin-bound paclitaxel compared with polyethylated castor oil-based paclitaxel in women with breast cancer. J Clin Oncol 23: 7794-7803, 2005.

35. Kurt M, Harputluoglu H, Dede DS, Gullu IH and Altundag K: Potential molecular targeted therapies in the management of the basal-like subtype of breast cancer. Breast 16: 111-112, 2007.

36. Finn RS, Dering J, Ginther C, et al: Dasatinib, an orally active small molecule inhibitor of both the src and abl kinases, selectively inhibits growth of basal-type/'triple-negative' breast cancer cell lines growing in vitro. Breast Cancer Res Treat 105: 319-326, 2007.

37. Quintas-Cardama A, Kantarjian H, Jones D, et al: Dasatinib (BMS-354825) is active in Philadelphia chromosome-positive chronic myelogenous leukemia after imatinib and nilotinib (AMN107) therapy failure. Blood 109: 497-499, 2007.

38. Christensen JG, Schreck R, Burrows J, et al: A selective small molecule inhibitor of c-Met kinase inhibits c-Met-dependent phenotypes in vitro and exhibits cytoreductive antitumor activity in vivo. Cancer Res 63: 7345-7355, 2003. 